\title{
Clinical features and natural history of clinically non-functioning pituitary incidentalomas
}

\author{
Pedro Iglesias ${ }^{1}$, Karina Arcano ${ }^{1}$, Vanessa Triviño ${ }^{2}$, Paula García-Sancho ${ }^{3}$, Juan José \\ Díez $^{1}$, Fernando Cordido ${ }^{2} \&$ Carles Villabona ${ }^{3}$
}

\author{
${ }^{1}$ Department of Endocrinology, Hospital Universitario Ramón y Cajal, Madrid, Spain; ${ }^{2}$ Department of \\ Endocrinology, Hospital Universitario A Coruña, A Coruña, Spain; ${ }^{3}$ Department of Endocrinology, Hospital \\ Universitario Bellvitge, Barcelona, Spain.
}

Objetive: To assess the clinical features of incidental clinically non-functioning pituitary adenoma (NFPA) and to analyze its natural history.

Methods: A multicenter retrospective study in patients with NFPA followed-up from 1992 to 2015 was performed.

Results: Fifty-seven patients were studied ( 29 women (50.9\%); age $55.8 \pm 16.7$ years. $43.9 \%$ were older than 60 years, $40.3 \%$ belonged to the age group of $40-60$ years and $15.8 \%$ were younger than 40 years. Most patients $(n=55,96.5 \%)$ were diagnosed by magnetic resonance imaging (MRI). $71.9 \%(n=41)$ were macroadenomas; 2 of them $(3.5 \%)$ were giant adenomas $(\geqq 4 \mathrm{~cm})$. Patients with macroadenomas were older than those with microadenomas $(59.5 \pm 16.7$ vs $46.4 \pm 18.1$ years, $P=0.007)$. Microadenomas were more common in women (41.3 vs $14.3 \%, P=0.023)$. About half of them $(n=28 ; 49.1 \%)$ showed suprasellar extension; of these, 19 (33.3\%) were accompanied by chiasmatic compression. Invasion of the cavernous sinuses was present in $28.1 \%(n=16)$. Hypopituitarism was present in $14(24.6 \%)$ patients. Twenty-four patients $(42.1 \%)$ underwent surgery. Twenty-six non-operated patients were evaluated after a median follow-up of 15.5 months (interquartile range, 5.7-32.7). No significant changes were found in the maximum tumor diameter at the end of follow-up $(1.2 \pm 0.6 \mathrm{vs} 1.2 \pm 0.7 \mathrm{~cm}$; NS). The majority of NFPAs evaluated $(n=23,88.5 \%)$ did not show any changes in size. In two cases $(7.7 \%)$ tumor size decreased and in one patient (3.8\%) increased.

Conclusion: Incidental NFPA is diagnosed by MRI preferably from the 5th decade of life without sex predilection. Most of them are macroadenomas, more commonly diagnosed in men and at an older age, compared to microadenomas. The suprasellar extension with chiasmatic compression and hypopituitarism are frequent at diagnosis. Most of the non-operated NFPAs remain with stable tumor size over time, being growth an unusual event. 